\title{
Corticosteroids, COVID-19 pneumonia, and acute respiratory distress syndrome
}

\author{
Michael A. Matthay ${ }^{1,2,3}$ and Katherine D. Wick ${ }^{1,3}$ \\ 'Cardiovascular Research Institute, 'Department of Medicine, and ${ }^{3}$ Department of Anesthesia, UCSF, San Francisco, California, USA.
}

\begin{abstract}
Although corticosteroids dampen the dysregulated immune system and sometimes are prescribed as an adjunctive treatment for pneumonia, their effectiveness in the treatment of coronavirus disease 2019 (COVID-19) remains controversial. In this issue of the $J C I$, Liu and Zhang et al. evaluated corticosteroid treatment in more than $\mathbf{4 0 0}$ patients with severe COVID-19. The authors assessed subjects retrospectively for cardiac and liver injury, shock, ventilation, mortality, and viral clearance. Corticosteroids in severe COVID-19-related acute respiratory distress syndrome (ARDS) were associated with increased mortality and delayed viral clearance. Here, we consider how to reconcile the negative effects of corticosteroids revealed by Liu and Zhang et al. with the favorable effects (reduced mortality) that were described in the RECOVERY trial. We posit that treatment timing, dosage, and COVID-19 severity determine immune response and viral outcome. Patients with moderate-to-severe COVID-19 pneumonia are likely to benefit from moderate-dose corticosteroid treatment when administered relatively late in the disease course.
\end{abstract}

\section{Corticosteroids in the} treatment of viral pneumonia

The effects of corticosteroids in the treatment of viral pneumonia and the acute respiratory distress syndrome (ARDS) have been the subject of controversy over decades, including several studies through the last 15 years. The majority of studies report a detrimental effect of corticosteroids for the treatment of influenza viral pneumonia, including studies during the H1N1 influenza outbreak of 2009-2010 (1, 2) and subsequent studies with $\mathrm{H} 5 \mathrm{~N} 1 \mathrm{influ}-$ enza pneumonia (3). Most studies were retrospective, though the analyses were adjusted for baseline differences between the corticosteroid-treated and untreated patients (4). During the 2003 severe acute respiratory syndrome (SARS) coronavirus outbreak, one retrospective study found evidence of benefit among patients with ARDS from SARS (5). Others showed either no benefit or evidence of harm (6). Notably, viral RNA clearance was delayed in patients who received early corticosteroids (7). In a retrospective study of corticosteroids in critically ill patients with Middle East respiratory syndrome, unadjusted mortality was higher among patients who received corticosteroids, and RNA viral clearance was delayed (8). Thus, the potential beneficial or harmful effect of corticosteroids in patients with SARS-coronavirus 2 (SARS-CoV-2) has been an area of major interest since the onset of the coronavirus disease 2019 (COVID-19) pandemic.

In this issue of the JCI, Liu and Zhang et al. performed a retrospective study of 774 patients with COVID-19-related acute respiratory insufficiency who developed ARDS in five tertiary care hospitals in China between December 2019 and

Related Article: p. 6417

Conflict of interest: MAM declares consultancy income from Citius Pharmaceuticals in 2020 for observational studies of ARDS and research support from Genentech-Roche for observational studies of ARDS.

Copyright: ( 2020 , American Society for Clinical Investigation.

Reference information: J Clin Invest. 2020;130(12):6218-6221. https://doi.org/10.1172/JCI143331.

March 2020 (9). Overall 28-day mortality was $38 \%$. Patients treated with corticosteroids $(n=409)$ had a significantly higher 28-day mortality than those without corticosteroid treatment $(n=365)$ (OR $1.77,95 \%$ CI $1.32-2.38, P<0.001)$, and corticosteroid use was an independent risk factor for mortality in a multivariate logistic regression analysis and an extended Cox regression model. Corticosteroids also delayed SARS-CoV-2 RNA clearance. Most patients did not present with ARDS at admission; $87 \%$ in the steroid group and $92 \%$ in the non-steroid group were on nasal cannula oxygen at admission, and their respiratory status deteriorated during hospitalization.

Notably, patients who received corticosteroids were substantially different from those who did not by several criteria. The steroid-treated group included fewer smokers, fewer patients with diabetes, and fewer patients with chronic cardiac or renal disease, but the baseline acute physiology and chronic health evaluation (APACHE) II score (severity of illness measure) was higher (12 vs. 10, $P=0.001)$. To account for these differences, a propensity analysis based on multiple variable regression was done that matched 182 patients who received steroids versus 182 patients who did not. The increased odds of death in patients treated with corticosteroids persisted among propensity-matched subjects $(\mathrm{OR}=1.64,95 \%$ CI 1.05-2.57, $P=0.032$ ) (9). This adjusted analysis reinforces the primary findings. However, it is still possible that the patients who received corticosteroids differed in some meaningful ways that cannot be fully captured by propensity matching. Traditionally, this is termed confounding by indication; in other words, the steroid-treated patients could have been sicker than patients not treated with steroids.

In contrast, the RECOVERY trial ( $n$ $=6425)$ reported a significant mortality benefit of corticosteroid therapy with 6 $\mathrm{mg}$ dexamethasone daily $(150 \mathrm{mg}$ hydrocortisone equivalent) for up to 10 days in 
A

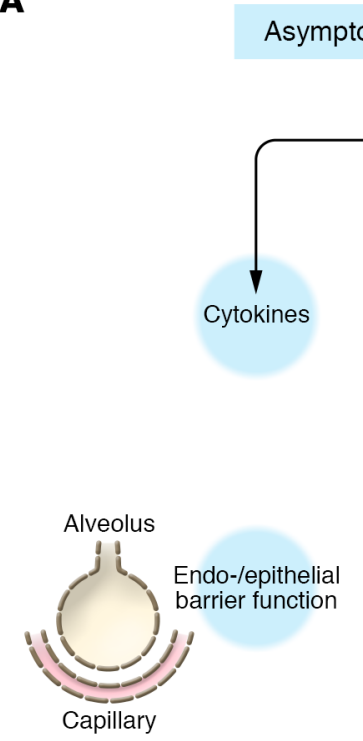

C

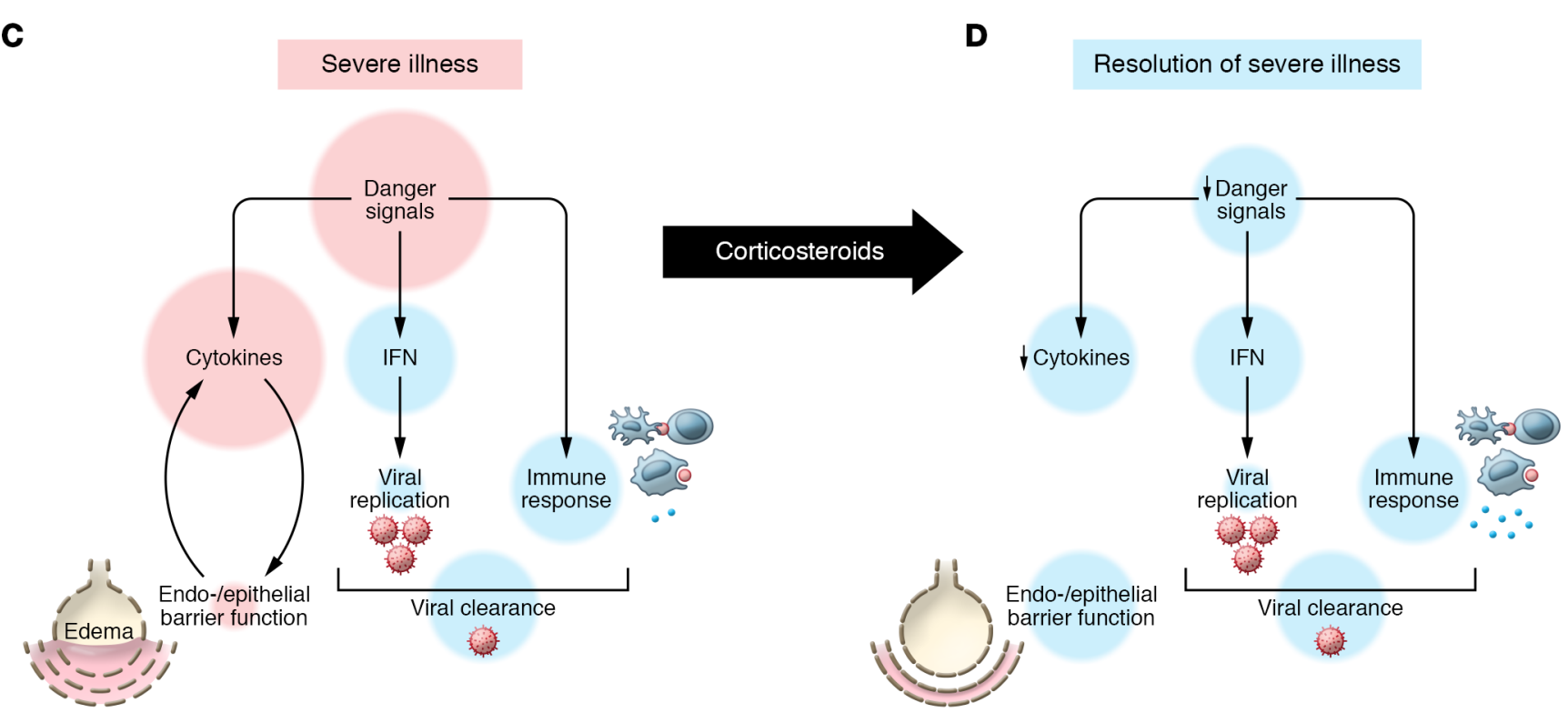

B

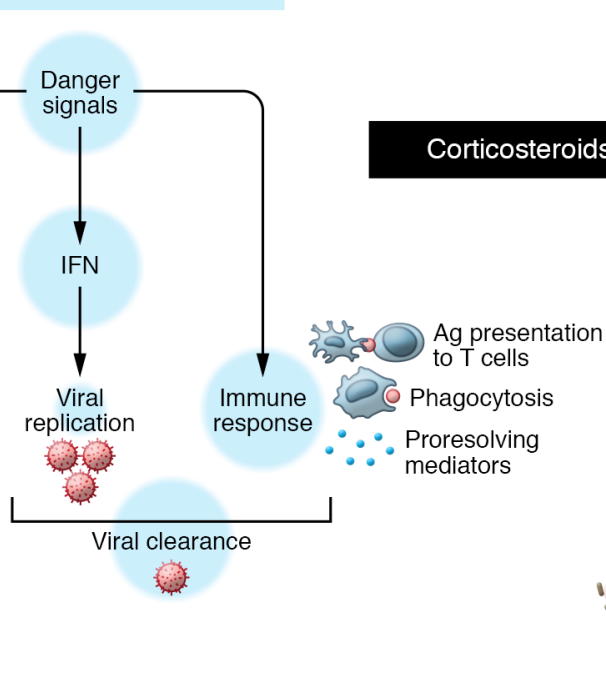

Worsening illness

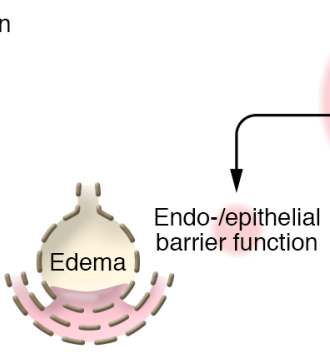

Danger

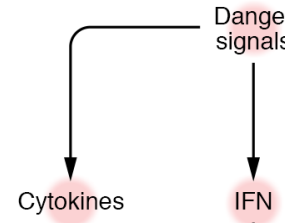

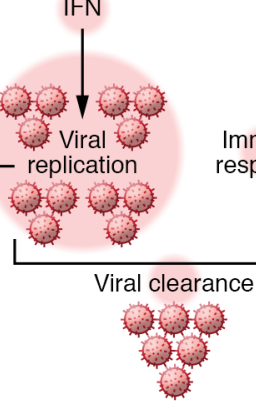

3 mmune $\bigcirc$ response
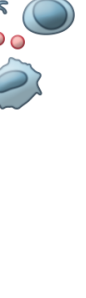

Figure 1. Model for deleterious or beneficial effects of corticosteroids in the treatment of COVID-19. (A) In asymptomatic or mild cases and in the absence of treatment, SARS-CoV-2 induces transcriptional upregulation of interferons (IFNs) and NF- $\kappa B$ activation, which promote cytokine production and activation of macrophages as well as demargination of PMNs. Antigens are presented to T cells and a targeted cytotoxic response ensues. (B) In worsening illness, corticosteroid treatment can delay pathogen recognition and control. Dampened danger signaling leads to impaired IFN release, unchecked viral replication, and consequent alveolar and lung damage. (C) In severe illness with COVID-19 without corticosteroid treatment, viral propagation to the alveoli amplifies danger signals and worsens alveolar epithelial and endothelial damage. Persistent damage leads to exuberant NF- $\mathrm{B}$ activation and inflammation worsens even as viral load decreases. (D) In severe cases of COVID-19 corticosteroid treatment may decrease proinflammatory cytokine burden and help resolution. Corticosteroids promote a proresolving macrophage phenotype that can clear cellular debris. Corticosteroids also reduce capillary permeability and increase alveolar edema fluid clearance, resulting in improved barrier function.

patients who were receiving oxygen therapy or mechanical ventilation, with the greatest benefit in mechanically ventilated patients (10). There was, however, a strong trend for greater mortality in patients who received dexamethasone and who were not requiring oxygen therapy. In a prespecified subgroup analysis, patients who received corticosteroids more than seven days after symptom onset had reduced 28-day mortality (rate ratio [RR] 0.69, 95\% CI 0.59-0.80), but there was no significant difference in mortality for the subgroup of patients who received steroids at or before seven days after symptom onset (RR 1.01, 95\% CI 0.87-1.17). Some patients were excluded because their treating physician determined that corticosteroids were con- traindicated, although the reasons for such a determination were not recorded. Also, there were no measurements of viral clearance in this pragmatic trial.

\section{Reconciling contradictory results}

Can the negative results of the Liu and Zhang et al. study (9) be reconciled with 
the favorable effects of dexamethasone on reducing mortality in the RECOVERY trial (10)? The timing of corticosteroid administration could be a major factor. In the Liu and Zhang et al. study, the vast majority of patients received corticosteroid therapy a median of three days before invasive mechanical ventilation, and the increase in mortality was driven by patients who received corticosteroids early in their hospitalization. Earlier corticosteroid administration might impair clearance of SARS-CoV-2, as suggested by delayed viral clearance in the steroid-treated patients. The dose of corticosteroid treatment is likely a second important factor. The median dose of corticosteroids in the study by Liu and Zhang et al. (9) was $200 \mathrm{mg}$ hydrocortisone equivalent, and nonsurvivors received a median of 400 mg. The detrimental effects of steroids on mortality were driven by patients in the higher-dose group, whereas there was no statistically significant difference in the odds of death in the lower-dose group. In contrast, patients in the RECOVERY group received the equivalent of $150 \mathrm{mg}$ hydrocortisone (10). A third factor is the severity of COVID-19. In the Liu and Zhang et al. study, there was no evidence of a deleterious effect of corticosteroid therapy in patients who were mechanically ventilated or in shock. The primary deleterious effect was in patients with less systemic organ failure (sequential organ failure assessment [SOFA] score < 7). Thus, the results from Liu and Zhang et al. (9) are consistent with a deleterious effect of corticosteroid therapy when administered to patients who are not as severely ill. These findings match reasonably well with the RECOVERY trial (10), in which corticosteroid administration was associated with different mortality rates by severity of illness.

\section{Mechanisms for steroid effects in COVID-19}

What are the molecular and cellular mechanisms by which steroids can have a detrimental or beneficial effect on viral pneumonia/ARDS and COVID-19? The normal host response to viral infection prompts transcriptional upregulation of interferons and activation of transcription factors such as nuclear factor kappa $B$ (NF- $\kappa \mathrm{B})$, stimulating the release of several cytokines to mobilize monocytes, macro- phages, lymphocytes, and neutrophils to respond to viral infection (11). Coronaviruses have developed various mechanisms to evade detection and targeting by the host response early after infection (12) Corticosteroid treatment in the early stage of viral infection can suppress host antiviral activity $(13,14)$, enhancing viral replication and cytopathic damage to the alveolar epithelial cells (15). Uptake of virus by the ACE-2 receptor on alveolar epithelial type 2 cells with enhanced cell injury and death would impair surfactant secretion (needed for alveolar inflation and stability) and inhibit vectorial alveolar fluid clearance, the main pathway for resolution of pulmonary edema (16). The degree of alveolar epithelial injury is a major determinant of the severity of ARDS (17). In contrast, corticosteroid therapy given to COVID-19 patients after the host has controlled viral replication could have a favorable effect by reducing proinflammatory cytokines, enhancing antiinflammatory cytokines and proresolving lipids, decreasing lung vascular permeability, improving epithelial barrier integrity, and promoting alveolar edema fluid clearance (18-20). We posit that there are potential deleterious and beneficial effects of corticosteroids at different stages of infection, lung injury, and ARDS (Figure 1).

In conclusion, the findings of Liu and Zhang et al. (9) taken together with those of the RECOVERY trial (10) suggest that patients with moderate-to-severe COVID-19 pneumonia likely benefit from moderate-dose corticosteroid treatment relatively late in the disease course, especially when patients require mechanical ventilation. Early treatment in milder disease seems harmful. Prospective studies are needed that include more data on SARS-CoV-2 viral shedding in the presence of both corticosteroid treatment and antiviral therapy with remdesivir (21) correlated with the specific level of oxygen support (nasal oxygen versus high-flow nasal oxygen) and ventilatory support (noninvasive ventilation versus invasive ventilation), and detailed information regarding comorbidities that may increase susceptibility to harm from corticosteroid treatment. The complex immune response to SARS-CoV-2 infection is still being fully characterized, including comprehensive lymphocyte profiles in patients with severe disease (22). Also, a recent study of COVID-19 patients indicated that severely and critically ill COVID-19 patients have impaired interferon responses that were associated with impaired viral clearance in the blood and an exaggerated inflammatory response driven in part by the transcription factor NF-кB (23). Ongoing studies of the immune activation pattern in patients with COVID-19 should provide additional insights into the timing and therapeutic effects of corticosteroids and help determine which COVID-19 patients will benefit or be harmed.

\section{Acknowledgments}

MAM acknowledges support by $\mathrm{NIH}$ grants HL123004, HL134828, and HL140026. KDW receives support from NIH grant 5T32GM008440-24.

Address correspondence to: Michael A. Matthay, 505 Parnassus Avenue, M-917, University of California, San Francisco, San Francisco, California 94143, USA. Phone:415.819.2807; Email: Michael. matthay@ucsf.edu.

1. Brun-Buisson C, Richard JC, Mercat A, Thiebaut AC, Brochard L. Early corticosteroids in severe influenza A/H1N1 pneumonia and acute respiratory distress syndrome. Am J Respir Crit Care Med. 2011;183(9):1200-1206.

2. Diaz E, et al. Corticosteroid therapy in patients with primary viral pneumonia due to pandemic (H1N1) 2009 influenza. J Infect. 2012;64(3):311-318.

3. Liem NT, et al. Clinical features of human influenza A (H5N1) infection in Vietnam: 20042006. Clin Infect Dis. 2009;48(12):1639-1646.

4. Moreno G, et al. Corticosteroid treatment in critically ill patients with severe influenza pneumonia: a propensity score matching study. Intensive Care Med. 2018;44(9):1470-1482.

5 . Chen RC, et al. Treatment of severe acute respiratory syndrome with glucosteroids: the Guangzhou experience. Chest. 2006;129(6):1441-1452.

6. Auyeung TW, et al. The use of corticosteroid as treatment in SARS was associated with adverse outcomes: a retrospective cohort study. JInfect. 2005;51(2):98-102.

7. Lee $\mathrm{N}$, et al. Effects of early corticosteroid treatment on plasma SARS-associated coronavirus RNA concentrations in adult patients. J Clin Virol. 2004;31(4):304-309.

8. Arabi YM, et al. Corticosteroid therapy for critically ill patients with Middle East respiratory syndrome. Am J Respir Crit Care Med. 2018;197(6):757-767.

9. Liu J, et al. Corticosteroid treatment in severe COVID-19 patients with acute respiratory distress syndrome. J Clin Invest. 2020;130(12):6417-6428. 
10. RECOVERY Collaborative Group, et al. Dexamethasone in hospitalized patients with Covid-19-preliminary report [published online May 19, 2020]. N Engl J Med. https://doi. org/10.1056/NEJMoa2021436.

11. McNab F, Mayer-Barber K, Sher A, Wack A, O'Garra A. Type I interferons in infectious disease. Nat Rev Immunol. 2015;15(2):87-103.

12. Kopecky-Bromberg SA, Martinez-Sobrido L, Frieman M, Baric RA, Palese P. Severe acute respiratory syndrome coronavirus open reading frame (ORF) 3b, ORF 6, and nucleocapsid proteins function as interferon antagonists. JVirol. 2007;81(2):548-557.

13. Cain DW, Cidlowski JA. Immune regulation by glucocorticoids. Nat Rev Immunol. 2017;17(4):233-247.

14. Miyata M, et al. Glucocorticoids suppress inflam- mation via the upregulation of negative regulator IRAK-M. Nat Commun. 2015;6:6062.

15. Qian Z, et al. Innate immune response of human alveolar type II cells infected with severe acute respiratory syndrome-coronavirus. Am J Respir Cell Mol Biol. 2013;48(6):742-748.

16. Matthay MA, Ware LB, Zimmerman GA. The acute respiratory distress syndrome. J Clin Invest. 2012;122(8):2731-2740.

17. Matthay MA, et al. Acute respiratory distress syndrome. Nat Rev Dis Primers. 2019;5(1):18.

18. Cain DW, Cidlowski JA. After 62 years of regulating immunity, dexamethasone meets COVID19. Nat Rev Immunol. 2020;20(10):587-588.

19.Perretti M, Ahluwalia A. The microcirculation and inflammation: site of action for glucocorticoids. Microcirculation. 2000;7(3):147-161.

20. Zizzo G, Hilliard BA, Monestier M, Cohen
PL. Efficient clearance of early apoptotic cells by human macrophages requires M2c polarization and MerTK induction. JImmunol. 2012;189(7):3508-3520.

21. Beigel JH, et al. Remdesivir for the treatment of COVID-19 - final report [published online October 8, 2020]. N Engl JMed. https://doi. org/10.1056/NEJMoa2007764.

22. Mathew $D$, et al. Deep immune profiling of COVID-19 patients reveals patient heterogeneity and distinct immunotypes with implications for therapeutic interventions [published online May 23, 2020]. bioRxiv. https://doi. org/10.1101/2020.05.20.106401.

23. Hadjadj J, et al. Impaired type I interferon activity and inflammatory responses in severe COVID-19 patients. Science. 2020;369(6504):718-724. 